\title{
Structure of the WFIKKN2 Follistatin domain and insight into GDF8/11 antagonism
}

Jason C. McCoy, Thomas B. Thompson

Email Contact: mccoyj5@mail.uc.edu

Growth Differentiation Factor 8 (GDF8), also known as Myostatin, is a member of the TGF- $\beta$ superfamily and is a potent negative regulator of skeletal muscle growth. Disruption of GDF8 signaling or injection of GDF8 inhibitors results in muscle hypertrophy. Muscle mass can also be stimulated through the upregulation of secreted GDF8 protein antagonists, such as Follistatin. In contrast, transgenic overexpression of GDF8 causes muscle wasting. Thus, in animals the balance of muscle mass is highly regulated by the appropriate levels of active GDF8, both during development and in the adult. Given that GDF8 plays a profound role on muscle biology there has been a strong interest in developing therapeutics that target GDF8 to alleviate muscle wasting in a variety of diseases or to treat cancer cachexia where tumors have been shown to upregulate GDF8. As such, anti-GDF8 therapeutics are within phase II and III clinical trials that directly target GDF8 or block signaling receptors. GDF8 is inhibited by a number of extracellular antagonists, one such antagonists, WFIKKN2, exhibits exceptional specificity for GDF8 and GDF11 (90\% identity). Similar to the less specific Follistatin family of antagonists, WFIKKN2 utilizes a number of small modular domains including at least one follistatin (FSD) domain. Although there is vast knowledge regarding the Follistatin family FSDs and how they contribute to GDF8 antagonism very little is known about how the WFIKKN2 FSD interacts with GDF8. To address this, we isolated the WFIKKN2 FSD and characterized its binding interaction with GDF8 and GDF11. In addition, we solved the crystal structure of the WFIKKN2 FSD to $1.39 \AA$. The structure revealed conserved, surface exposed, hydrophobic residues that when mutated to alanine reduced full-length WFIKKN2 antagonism of GDF8. Comparison of the WFIKKN2 FSD to follistatin and FSTL3 indicates that WFIKKN2 utilized a different surface of the FSD to interact with GDF8. Further comparison reveals drastic differences in the domain arrangement classified as either an open or closed conformation. Collectively, the results highlight that although FSDs have conserved domain architecture they exhibit unique functions in biology. 